\title{
25 Research Soure \\ Effects of Co-infection With Clonorchiasis Sinensis on T Cell Exhaustion Levels in Patients With Chronic Hepatitis B
}

\section{Huimin Dong}

Third Affiliated Hospital of Sun Yat-Sen University

\section{Minqi Luo}

Third Affiliated Hospital of Sun Yat-Sen University

\section{Mei Shang}

Third Affiliated Hospital of Sun Yat-Sen University

\section{Yang Wang}

Third Affiliated Hospital of Sun Yat-Sen University

\section{Yuechun Fu}

Third Affiliated Hospital of Sun Yat-Sen University

Bo Hu ( $D$ hubo@mail.sysu.edu.cn )

Third Affiliated Hospital of Sun Yat-Sen University

\section{Research}

Keywords: Clonorchiasis Sinensis, Chronic hepatitis B, T cell exhaustion, Inhibitory receptors, Suppressive cytokines

Posted Date: August 18th, 2021

DOl: https://doi.org/10.21203/rs.3.rs-789633/v1

License: (c) (i) This work is licensed under a Creative Commons Attribution 4.0 International License. Read Full License 


\section{Abstract}

\section{Background}

To investigate the effects of co-infection with $\mathrm{C}$. sinensis on $\mathrm{T}$ cell exhaustion levels in patients with chronic hepatitis $B$

Methods

Inhibitory receptors and suppressive cytokines expression in circulating CD4+ and CD8+ T cells was detected by flow cytometry. The correlations between PD-1 and TIM-3 expression and alanine aminotransaminase (ALT) levels, aspartate aminotransferase (AST) levels and HBV DNA levels were analyzed using GraphPad Prism 7.0.

Results

PD-1 and TIM-3 expression levels were significantly higher on CD4+T and CD8+T cells from co-infected patients than on those from the HBV patients. In addition, CD4+T cells and CD8+T cells function was inhibited by C. sinensis and HBV coinfection, secreting low levels of IFN- - , IL-2 and TNF-a. Then, a significant positive correlation was found between the PD-1 and TIM-3 expression levels on T cells and the AST ,ALT levels and HBV DNA levels.

\section{Conclusions}

Our current results suggested that $C$. sinensis co-infection could exacerbate $T$ cell exhaustion in patients with chronic hepatitis B. Furthermore, it maybe one possible reason for the weaker response to antiviral therapies and the chronicity of HBV infection in co-infected patients. We must realize that the importance of C.sinensis treatment for HBV infected patients. It might provide useful information for the clinical doctors to choose the right treatment plans.

\section{Background}

Clonorchiasis, resulted from Clonorchis sinensis (C. sinensis), is a food-borne

parasitic disease. Over 35 million people are infected by C. sinensis in Asia, among

which about 15 million infected people were prevalent in China[1-3]

.One third of them are from Guangdong province[4].Clonorchis sinensis is a fish-borne trematode that locates in the bileduct of mammals including humans[5]. Freshwater fish act as the intermediate hosts in which the cercariae penetrate and transform to the metacercariae. Humans become infected by eating raw or undercooked freshwater fish[6]. Clinically, clonorchiasis patients accompany different severity of the symptoms. Most of the infected people are not treated in time because of mild or unspecific symptoms, such as asthenia, nausea,diarrhea, jaundice, hepatomegaly and liver tenderness. So, 
Clonorchiasis is a major but surprisingly neglected tropical disease in China. Only about $10 \%$ of the infected patients who have been treated in time show obvious acute clinical symptoms in the early infection, such as cholelithiasis, cholangitis and cholecystitis.What's worse, chronic infection can develop to the cirrhosis or cholangiocarcinoma[7-9].The excretory-secretory products (ESPs) of C.Sinensis which cause chemical damage often trigger highly polarized immune responses during chronic infections[1012]. Meanwhile, our previous research results show that the ESPs could induce immune cells to mainly produce Th2 cytokines such as IL-4, IL-6 and IL-10[13-15].

Hepatitis B virus (HBV) infection is a major public health challenge that may cause severe complications, such as cirrhosis and hepatocellular carcinoma (HCC)[13-15]. Adaptive immune responses against HBV, including CD4 + T and CD8 + T cells, are thought to play a dominant role in determining whether HBV infection is followed by recovery or viral persistence[17-19].A lot of studies have indicated the essentiality of $\mathrm{T}$ cell exhaustion in HBV infection[20-23]. Patients who infected with acute hepatitis B presented progressive high frequency and functional impairment peripheral blood[24].As the same time, experimentally infected chimpanzees verified the antiviral functions of CD8 $+T$ cells, indicating that CD8 $+T$ cells that were knocked out can delayed the clearance of virus. On contrast, chronic HBV infection often shows weak virus specific CD $8+T$ cell reactivity and then arises a inability state of $T$ cell which is described as the "exhaustion" $[25,26]$. T cell exhaustion was firstly described during chronic lymphocytic choriomeningitis virus (LCMV) infection in mice more than a decade ago as dysfunction of antigenspecific T cells[27, 28]. Since then, $T$ cell exhaustion has been confirmed in plenty of animal models and in humans with chronic viral infections and cancer[27, 28]. T cell exhaustion is characterized by expression of multiple cell surface inhibitory receptors, secretion suppressive cytokines and appearance of immunoregulatory cells, such as programmed cell death-1(PD-1), lymphocyte activation gene-3(LAG3), T cell immunoglobulin mucin-3 (Tim-3), cytotoxic T lymphocyte-associated antigen-4 (CTLA-4), IL-2, IFN- $\gamma$ and TNF-a[30-34]. HBV can't be cleaned up and continuous replication, then develop into liver cirrhosis and liver cancer. The main reason for the chronicity of HBV infection is virus specific $T$ cell exhaustion. Reversal of these exhausted T cells will pave the way for the development of more effective immunotherapeutic strategies for the treatment of chronic HBV infection.

Some studies had shown that in areas where $\mathrm{C}$. sinensis is prevalent, such as guangdong province, we often observe the concurrent infection of HBV and C. sinensis. And both C. sinensis infection and chronic hepatitis B virus infection can cause liver diseases. There were few studies on the co-infection with HBV and $C$. sinensis. The aim of our study is to evaluate the effect of co-infection with $C$. sinensis on $T$ cell exhaustion levels in patients with chronic hepatitis $\mathrm{B}$. Our results showed that $\mathrm{C}$. sinensis co-infection could exacerbate $T$ cell exhaustion in patients with chronic hepatitis B. Furthermore, it maybe one possible reason for the weaker response to antiviral therapies in co-infected patients. We must realize that the importance of C.sinensis treatment for HBV infected patients. It might provide useful information for the clinical doctors to choose the right treatment plans.

\section{Methods}




\section{Subjects}

All patients were hospitalized or followed-up in the Third Affiliated Hospital of Sun Yat-sen University. According to the infection of HBV and $\mathrm{C}$. sinensis, the patients were divided into three groups: patients who were mono-infected with HBV surface-antigen (HBsAg)-positive and HBV DNA $>20 \mathrm{IU} / \mathrm{mL}$,patients who were mono-infected with $\mathrm{C}$. sinensis eggs-positive and patients who were co-infected with HBsAgpositive, HBV DNA $>20 \mathrm{IU} / \mathrm{mL}$ and C. sinensis eggs-positive. The exclusion criterion for patients with the following other causes of chronic liver damage were excluded from the study: hepatitis A, C, D and E, HIV, Schistosoma japonicum, Schistosoma mansoni or other parasites,alcoholic or autoimmune factor. And Patients with diabetes, hematological system diseases, serious heart diseases, pregnant women were also excluded from the study. Twenty-five healthy individuals with matched age and sex were also enrolled as normal controls. We summarizes the clinical characteristics obtained for the enrolled subjects in Table 1. The study protocol was approved by the Clinical Research Ethics Committee of the Third Affiliated Hospital, Sun Yat-sen University, Guangzhou, China and informed consent forms were signed by all participants according to the Declaration of Helsinki. 
Table 1

Clinical Characteristics of the Study Groups

\begin{tabular}{|c|c|c|c|c|}
\hline & $\begin{array}{l}\text { HC group } \\
(n=25)\end{array}$ & $\begin{array}{l}\text { HBV group } \\
(n=24)\end{array}$ & $\begin{array}{l}\text { C. sinensis } \\
\text { group } \\
(n=23)\end{array}$ & $\begin{array}{l}\text { co-infected group } \\
(n=20)\end{array}$ \\
\hline $\begin{array}{l}\text { Age,years } \\
(\text { Mean } \pm S D)\end{array}$ & $45.48 \pm 11.34$ & $43.29 \pm 12.35$ & $53.17 \pm 11.58$ & $45.90 \pm 7.52$ \\
\hline Gender(male/female) & $19 / 6$ & $18 / 6$ & $19 / 4$ & $18 / 2$ \\
\hline $\mathrm{HBsAg}(\mathrm{COI})$ & negative & $\begin{array}{l}5390.20 \pm \\
3397.34\end{array}$ & negative & $\begin{array}{l}4893.50 \pm \\
2892.48\end{array}$ \\
\hline $\begin{array}{l}\text { HBV DNA log10 } \\
\text { copies/ml(Mean } \pm \text { SD ) }\end{array}$ & negative & $4.73 \pm 1.99$ & negative & $5.50 \pm 1.92$ \\
\hline $\operatorname{ALT}(\mathrm{U} / \mathrm{L})$ & $\begin{array}{l}17.00(8.00- \\
34.00)\end{array}$ & $\begin{array}{l}48.50(13.00- \\
774.00)\end{array}$ & $\begin{array}{l}45.00(10.00- \\
296.00)\end{array}$ & $\begin{array}{l}455.50(18.00- \\
894.00) \text { abc }\end{array}$ \\
\hline $\mathrm{AST}(\mathrm{U} / \mathrm{L})$ & $\begin{array}{l}17.00(10.00- \\
40.00)\end{array}$ & $\begin{array}{l}44.50(16.00- \\
660.00)\end{array}$ & $\begin{array}{l}39.00(12.00- \\
172.00)\end{array}$ & $\begin{array}{l}243.50(20.00- \\
902.00) \text { abc }\end{array}$ \\
\hline $\mathrm{TB}(\mu \mathrm{mol} / \mathrm{L})$ & $\begin{array}{l}8.30(4.50- \\
15.80)\end{array}$ & $\begin{array}{l}21.35(4.70- \\
478.60)\end{array}$ & $\begin{array}{l}12.50(4.00- \\
382.10)\end{array}$ & $\begin{array}{l}88.60(6.90- \\
469.50) a b c\end{array}$ \\
\hline \multicolumn{5}{|c|}{$\begin{array}{l}\text { Data are expressed as the Mean } \pm \text { SD or median and ranges. AST: aspartate aminotransferase; ALT: } \\
\text { alanine aminotransferase; HBV: hepatitis B virus; TB: total bilirubin }\end{array}$} \\
\hline \multicolumn{5}{|c|}{ a Statistically significantly different vs. healthy control group } \\
\hline \multicolumn{5}{|c|}{ b Statistically significantly different vs. HBV mono-infected group } \\
\hline \multicolumn{5}{|c|}{ c Statistically significantly different vs. C. sinensis mono-infected group } \\
\hline A & & & & \\
\hline
\end{tabular}

\section{Virological and Biochemical Assessments}

Biochemical assessments was measured using automated biochemical techniques (Hitachi 7600, Tokyo, Japan). The serum HBsAg level was detected using the electrochemiluminescence immunoassay kit for the COBAS-e801 system (Roche Diagnostics, Mannheim, Germany), with a positive result recorded as COI $>1.00$.The serum levels of HBV DNA were determined by real-time PCR with a lower detection limit of 20 IU/mL(Roche Diagnostics, Mannheim, Germany)

\section{Peripheral Blood Mononuclear Cells Isolation}

Peripheral Blood Mononuclear Cells(PBMCs) were from fresh heparinized blood $(5 \mathrm{~mL})$ collected from each group patient and isolated by Ficoll-Hypaque density gradient centrifugation (HaoYang Biological Manufacture, Tianjin, China). PBMCs were cultured at $1 \times 106$ cells/mL in RPMI 1640 (Invitrogen Gibco, USA) supplemented with $10 \%$ fetal bovine serum (FBS; Invitrogen Gibco). 


\section{Determination of PD-1,TIM-3,LAG-3 and CTLA-4 expression by flow cytometry}

The PBMCs were washed twice with PBS buffer and incubated with Anti-CD3-PE-cy7(Bioscience, USA), anti-CD4-APC(Bioscience), anti-CD8-FITC (Bioscience), anti-PD-1-PerCP-cy5.5(Bioscience), anti-Tim-3PE(Bioscience), anti-LAG-3-A1eXa FluorX647(Bioscience), and anti-CTLA-4-BV421(Bioscience) for surface staining at room temperature for $30 \mathrm{~min}$ in the dark. IgG isotype control antibodies were used as the negative controls.Staining cells were analyzed with a BD FACS Aria II analyzer (BD Biosciences) and data was analyzed with FlowJo version 10 for Windows(Tree Star Inc, USA).

\section{Determination of intracelluar cytokine release by flow cytometry}

The PBMCs were stimulated with a cell stimulation cocktail (1:500, eBioscience, USA). After 4-6h of incubation, anti-CD3-PE-cy7(Bioscience, USA), anti-CD4-APC(Bioscience) and anti-CD8-FITC (Bioscience) was used for cells staining at room temperature for $30 \mathrm{~min}$ in the dark. After permeabilization and fixation, the cells were washed twice with PBS buffer and incubated with anti-IFN- $\gamma$-Alexa Fluor 647(Biolegend), anti-IL-2-BV42(Biolegend) and anti-TNF-a-PE(Biolegend) at room temperature for 30 min in the dark. IgG isotype control antibodies were used as the negative controls.Staining cells were analyzed with a BD FACS Aria II analyzer (BD Biosciences) and data was analyzed with FlowJo version 10 for Windows(Tree Star Inc, USA).

\section{Statistical analysis}

All data of continuous variables were presented as the mean values \pm standard error or median and ranges. Differences in continuous variables were performed by independent-samples $T$ test or ANOVA for comparison with more than two groups. The Wilcoxon rank sum test was used for non-parametric data. The correlations between the PD-1,TIM-3, LAG-3 and CTLA-4 expression levels and the ALT, AST, TB, HBsAg and HBV DNA levels were analyzed in the co-infected group by Pearson's correlation analysis. The data were analyzed using GraphPad Prism 7.0. P values $<0.05$ were considered statistically significant.

\section{Results}

\section{Demographic and clinical characteristics of the study subjects}

The enrolled subjects in this study were classified into four groups: co-infected group $(n=20), H B V$ group $(n=24), C$. sinensis group $(n=23)$, and HC group $(n=25)$. Age, gender, liver function, HBsAg and serum HBV DNA concentrations were demonstrated in Table 1.The co-infected group showed higher levels of ALT, AST and TB than the C. sinensis group and the HC group. Especially, the levels of ALT, AST and TB in the co-infected group was significantly higher than that in the HBV group $(p<0.05$, respectively) 
.However, no difference in the HBV DNA log copies and HBsAg was found in the co-infected group and in the HBV group $(P>0.05)$. In brief, these data indicate that $C$. sinensis may weak liver function and aggravate the disease state in patients with chronic hepatitis B. Eventually, C. sinensis and HBV coinfection may lead to the chronicity of HBV infection.

Expression of inhibitory receptors on the surface of peripheral blood CD4 + T and CD8 + T cells from the co-infected group, HBV group and C. sinensis group and the HC group.

The frequencies of $C D 4+T$ and $C D 8+T$ cells with surface expression of the inhibitory receptors PD1,TIM-3, LAG-3 and CTLA-4 were evaluated in the co-infected group, HBV group, C. sinensis group and HC group using flowcytometry. Compared with HC group, the percentage of PD- $1+\mathrm{CD} 4+\mathrm{T}$ cells, PD-1 + CD8 + T cells, TIM-3 + CD $4+T$ cells, TIM-3 + CD $8+T$ cells , LAG-3 + CD $8+T$ cells, CTLA-4 + CD $4+T$ cells and CTLA-4 CD8 $+T$ cells were all significantly increased in both HBV group and Co-infected group (all $\mathrm{P}<$ 0.002 , Fig. 1), while no significant difference was found in the LAG-3 on the CD $4+T$ cells.

(Fig. 1D).Significantly higher frequencies of PD-1 + CD4+, PD-1 + CD8+, TIM-3 + CD 4 + and TIM-3 + CD 8 + cells were observed in the co-infected group than in the HBV group $(P=0.000, P=0.045, P=0.006$, and $P$ $=0.010$, respectively). No difference was found in the LAG-3 and CTLA-4 expression levels

(Fig. 1D,E,H,I).This result demonstrated that high PD-1 and TIM-3 expression in the co-infected group could exacerbate $T$ cell exhaustion in patients with chronic hepatitis $B$.

Difference in suppressive cytokines production between the CD4 + T and CD8 + T cells from the Coinfected group, HBV group and C. sinensis group and the HC group.

The percentages of CD $4+T$ and CD $8+T$ cells with suppressive cytokine expression, such as IL-2, IFN- $\gamma$, and TNF-a were assessed in the four group by flow cytometry.

Compared with HC group, the percentage of IFN-y, IL-2 and TNF- $\alpha$ in both the CD $4+T$ cells and CD $8+T$ cells were all significantly decreased in both HBV group and co-infected group (all $P<0.001$, Fig. 2 ), and lower IL-2 expression levels were detected in both the CD $4+T$ cells and CD $8+T$ cells obtained from the co-infected group than those in the CD4 $+T$ cells and $C D 8+T$ cells obtained from the HBV group $(P=$ $0.004, P=0.016$, Fig. 2B,2E). The co-infected group showed lower levels of IFN- $y$ and TNF- $a$ in the CD8 + T cells than the HBV group $(P=0.032, P=0.038$, Fig. $2 F, 2 G)$, while no significant difference was found in the IFN- $y$ and TNF- $a$ in the CD $4+T$ cells. (Fig. 2C,2D). These findings suggested that CD $4+T$ cells and $\mathrm{CD} 8+T$ cells function was inhibited by $\mathrm{C}$. sinensis and HBV coinfection, secreting low IFN- $y$, IL-2 and TNF-a.

Correlation between clinical indicators and the surface expression of the inhibitory receptors expression levels on T cells

Since PD-1 and TIM-3 were highly expressed by CD $4+T$ cells and CD8 + T cells in co-infected group relative to the expression levels in the HBV group, we analyzed the association between the PD-1 and TIM-3 expression levels on T cells and the serum 
ALT levels ,AST levels, TB levels, the HBSAg levels and the HBV DNA levels among the co-infected patients. Our results demonstrated that there were significant positive correlations between the levels of ALT and PD-1 and TIM-3 expression on CD4+ (PD-1 + CD4+: $r=0.696 \mathrm{P}=0.001, \mathrm{TIM}-3+\mathrm{CD} 4+: \mathrm{r}=0.600, \mathrm{P}$ $=0.005$, Fig. $3 A, C)$ and CD8 $+T$ cells $(P D-1+C D 8+: r=0.498, P=0.026, T I M-3+C D 8+: r=0.575, P=0.008$, Fig. $3 E, G$ ) among the co-infected patients. A positive correlation was observed between PD-1 and TIM-3 expression and the serum AST level on CD4+ (PD-1 + CD4+: $r=0.716, P=0.000, T I M-3+C D 4+: r=0.524$, $P=0.018$ Fig. $3 B, D)$ and CD $8+T$ cells $(P D-1+C D 8+: r=0.628, P=0.003, T I M-3+C D 8+: r=0.564, P=$ 0.010 , Fig. $3 F, H)$ among the co-infected patients. Meanwhile, there was a significant positive correlation between HBV DNA levels and PD-1 and TIM-3 expression on CD4+ (PD-1 + CD4+: $r=0.514, P=0.020$, TIM$3+\mathrm{CD} 4+: \mathrm{r}=0.501, \mathrm{P}=0.025$, Fig. 3Q, $R$ ) and CD8 + T cells (PD-1 + CD8+: $r=0.527, P=0.017, \mathrm{TIM}-3+$ $C D 8+: r=0.539, P=0.014$, Fig. $3 S, T$ ) among the co-infected patients. However, no association was found between the PD-1 and TIM-3 expression and TB levels on CD4+ (PD-1 + CD4+: $r=0.086, P=0.718, T I M-3$ $+C D 4+: r=0.176, P=0.457$, Fig. 3l,J) and CD8 + T cells ( PD-1 + CD8+: $r=0.131, P=0.582, T I M-3+C D 8+:$ $r=0.222, P=0.347$, Fig. $3 K, L)$ and there was no correlation between PD-1 expression and HBSAg levels on CD4+ (PD-1 + CD4+: $r=-0.023, P=0.923, T I M-3+C D 4+: r=-0.089, P=0.708$, Fig. 3M,N) and CD8 + T cells (PD-1 + CD8+: $r=-0.198, P=0.403$, TIM-3 + CD8+: $r=0.179, P=0.451$, Fig. 30,P) in the co-infected patients. These data suggested that the inhibitory receptors PD- 1 and TIM-3, highly expressed by CD $4+T$ cells and $\mathrm{CD} 8+\mathrm{T}$ cells from the co-infected group, were related to the degree of hepatic injury and high viral replication.

\section{Discussion}

In our study, we investigated the relationship between HBV infection and C. sinensis infection and further provided the evidence that Clonorchis sinensis co-infection could exacerbate $T$ cell exhaustion in patients with chronic hepatitis $B$.

In this study, we focused on the exhausted T cells, especially those in patients with co-infection C. sinensis and HBV. Actually, T cell exhaustion results in inhibition of host immune responses, which is a status of gradual T cell dysfunction that arises during chronic infections[35]. So immunoregulation is centrally involved. The main performances can be grouped into three categories: cell surface inhibitory receptors, cell factors and immunoregulatory cell types[36]. Our data showed that the cell surface inhibitory receptors such as PD-1, TIM-3, LAG-3 and CTLA-4 levels were significantly higher expressed by CD8 $+\mathrm{T}$ cells in CHB group than in HC group. CD $4+T$ cells expressed higher PD-1,TIM-3 and CTLA-4 levels, not including LAG-3, in CHB group than in HC group. In addition, we also analyzed the PD-1,TIM-3, LAG-3 and CTLA-4 expression levels on both CD $4+T$ cells and CD $8+T$ cells among the $C$. sinensis monoinfected patients. These inhibitory receptors were higher expressed in the $\mathrm{C}$. sinensis group relative to the expression levels in the HC group.On the other hand, lower IFN- $\gamma$, IL-2 and TNF-a expression levels were detected in both the CD4 + T cells and CD $8+T$ cells from both $C H B$ group and $C$. sinensis group than in the $\mathrm{T}$ cells obtained from the $\mathrm{HC}$ group.Our results suggest that the two infectious factors of hepatitis $B$ and $\mathrm{C}$. sinensis would eventually lead to the T cell exhaustion. 
Hepatitis B virus (HBV) is the most prevalent virus that leads to liver inflammation and injury. The ability to clear HBV after infection is associated with a strong specific $T$-cell response, and during chronic infections the function of exhausted T cells is often characterized with varying degrees of damage[37, 38]. C.sinensis always activate greatly polarized immune responses, especially during chronic infection. During HBV infection and clearance, It is well known that CD8 + T cell play a key role. However, CD $4+T$ cells are the key factor regulating on the cellular CTL response to HBV [39]. According to previous research, the lack of CD4 + T cells was the main cause of CD8 $+T$ cell exhaustion[40]. Like CD8 + T cells, specific CD $4+T$ cells also lose effector function during chronic viral infection $11-14$. Therefore longer persistent infection or loss of help from CD4 + T cells leads to more severe T cell exhaustion[41].Although $T$ cell exhaustion $C D 4+T$ cells have remained important, few researches about $C D 4+T$ cell exhaustion in chronic HBV-infected patients are mentioned $[42,43]$. These may be the reason that our study focused on both CD $4+T$ cells and CD $8+T$ cells.

As we known, it will be chronic HBV infection, when the virus can't be cleaned up and continuous replication,then develop into liver cirrhosis and liver cancer. The main reason for the chronicity of HBV infection is virus specific T cell exhaustion[24]. C. sinensis co-infection could exacerbate T cell exhaustion in patients with chronic hepatitis B.Our results were consistent with this findings. Our data showed that PD-1 and TIM-3 levels were significantly higher expressed on CD $4+T$ cells and CD $8+T$ cells in the co-infected group than those in the HBV group. At the same time, IFN- $y$, IL-2 and TNF-a expression levels were significantly lower detected in the CD8 $+T$ cells from co-infected group than those in the CD8 $+T$ cells obtained from the HBV group. But in CD4 + T cells, only IL-2 expression levels were significantly lower detected from co-infected group than those from the HBV group. This is consistent with the previous notion that in co-infected patients the efficacy of anti-viral treatment was better in patients who were prescribed with entecavir and praziquantel than entecavir alone[44]. This result further indicated that the efficacy of HBV antiviral treatment was related to the removal worms in co-infected patients. One possible reason for the weaker response to antiviral therapies in co-infected patients was the $T$ cell exhaustion.

Many cell surface inhibitory receptors can coregulate T cell exhaustion. T cells during chronic infection in animal models and in humans can also express PD-1, LAG-3, TIM-3, CTLA-4 and many other inhibitory receptors[10-12]. The pattern of inhibitory receptor expressed on the T cell can substantially affect the severity of dysfunction[45]. Our results are consistent with these findings. Furthermore, the recovery of function is increased considerably by simultaneous block of the PD-1 pathway, LAG-3 pathway, CTLA-4 pathway and TIM-3 pathway[46-48]. Our data showed that only PD-1 and TIM-3 levels were significantly higher expressed on T cells in the co-infected group than those in the HBV group. No difference was found in the LAG-3 and CTLA-4 expression levels. Further investigations are required to address this point. It may be due to the small number of these cases.

T cells mainly secrete suppressive cytokines such as IL-2, IFN- $\gamma$ and TNF-a to eradicate viruses and parasites causing intracellular infections and play an important role in cellular immunity[49]. Furthermore, 
we sought to understand the T cell exhaustion, as measured by changes in the IFN- $\gamma$, IL-2 and TNF-a secretion.

These findings suggested that $\mathrm{CD} 4+\mathrm{T}$ cells and $\mathrm{CD} 8+\mathrm{T}$ cells function was inhibited by $\mathrm{C}$. sinensis and HBV coinfection, secreting lower IFN- $y$, IL-2 and TNF-a, and these suppressive cytokines expression levels were significantly lower detected in the CD8 $+T$ cells from co-infected group than those in the CD $8+T$ cells obtained from the HBV group. Obviously, C.sinensis has more effect on CD8 + T cells from coinfected group.

Moreover, we observed that a significant positive correlation between the levels of PD-1 and TIM-3 expression either on CD4 and CD8 T cells with the conventional markers for hepatic injury. This result demonstrated that the upregulation of PD-1 and TIM-3 expression were positively correlated with the level of hepatic injury and high viral replication. Because both ALT and AST are the laboratory common indicators used in monitoring the progression of hepatic injury[50], the conclusions of this correlation further suggest that PD-1 and TIM-3 may be some novel indicators indirectly reflecting the persistence of chronic HBV and C. sinensis coinfection.

\section{Conclusions}

In summary, this study is the first to provide strong evidence that $C$. sinensis co-infection could exacerbate T cell exhaustion in patients with chronic hepatitis B. C. sinensis and HBV coinfection may lead to the chronicity of $\mathrm{HBV}$ infection, and $\mathrm{C}$. sinensis may play a role in the unresponsiveness to antiviral therapy in co-infected patients. We must realize that the importance of C.sinensis treatment for HBV infected patients.It might provide useful information for the clinical doctors to choose the right treatment plans.

\section{Abbreviations}

C. Sinensis: Clonorchiasis Sinensis; PD-1: Programmed death-1; TIM-3: T cell immunoglobulin domain and mucin domain-3 ; LAG-3: lymphocyte activation gene-3; CTLA-4: Cytotoxic T-Lymphocyte Associated Protein 4; ALT: alanine aminotransaminase; AST: aspartate aminotransferase; TB: total bilirubin囚PBMCs: Peripheral Blood Mononuclear Cells; ESPs: excretory-secretory products ; HBV: Hepatitis B virus

\section{Declarations}

\section{Acknowledgements}

We are thankful to all volunteer who participated in this study.

Authors' contributions 
HD designed the study and wrote the manuscript. ML, YW and YF collected the samples and performed the experiments. MS analyzed and interpreted the results. BH reviewed and edited the manuscript. All authors read and approved the final manuscript.

\section{Funding}

This work was supported by grants from the National Natural Science Foundation of China (81902082,81802403).

\section{Availability of data and materials}

Not applicable.

\section{Ethics approval and consent to participate}

The study protocol was approved by the Clinical Research Ethics Committee of the Third Affiliated Hospital, Sun Yat-sen University, Guangzhou, China (permission number 0204501) and informed consent forms were signed by all participants according to the Declaration of Helsinki.

\section{Consent for publication}

Not applicable.

\section{Competing interests}

The authors declare that they have no competing interests.

\section{References}

1. Qian MB, Utzinger J, Keiser J, Zhou XN. Clonorchiasis. Lancet. 2016;387:800-10.

2. Na BK, Pak JH, Hong SJ. Clonorchis sinensis and clonorchiasis. Acta Trop. 2020;203:105309.

3. Deng ZH, Fang YY, Zhang QM, Mao Q, Pei FQ, Liu MR. The control of clonorchiasis in Guangdong province, southern China. Acta Trop. 2020;202:105246.

4. Wang D, Young ND, Korhonen PK, Gasser RB. Clonorchis sinensis and Clonorchiasis: The Relevance of Exploring Genetic Variation. Adv Parasitol. 2018;100:155-208.

5. Young ND, Campbell BE, Hall RS, Jex AR, Cantacessi C, Laha T, et al. Unlocking the transcriptomes of two carcinogenic parasites, Clonorchis sinensis and Opisthorchis viverrini. PLoS Negl Trop Dis. 2010;4:e719.

6. Liang C, Hu XC, Lv ZY, Wu ZD, Yu XB, Xu J, et al. [Experimental establishment of life cycle of Clonorchis sinensis]. Zhongguo Ji Sheng Chong Xue Yu Ji Sheng Chong Bing Za Zhi. 2009;27:148-50. 
7. Qian MB, Chen YD, Liang S, Yang GJ, Zhou XN. The global epidemiology of clonorchiasis and its relation with cholangiocarcinoma. Infect Dis Poverty. 2012;1:4.

8. Tang $Z L$, Huang $Y, Y u X B$. Current status and perspectives of Clonorchis sinensis and clonorchiasis: epidemiology, pathogenesis, omics, prevention and control. Infect Dis Poverty. 2016;5:71.

9. Choi BI, Han JK, Hong ST, Lee KH. Clonorchiasis and cholangiocarcinoma: etiologic relationship and imaging diagnosis. Clin Microbiol Rev. 2004;17:540-52.

10. Nagano I, Wu Z, Takahashi Y. Functional genes and proteins of Trichinella spp. Parasitol Res. 2009;104:197-207.

11. Santra A, Chowdhury A, Ghosh A, Mazumder DN. Development of an animal model of hepatic fibrosis by excretory-secretory antigen of Ascaris suum. Indian J Gastroenterol. 2000;19:119-21.

12. Xu Y, Chen W, Bian M, Wang X, Sun J, Sun H, et al. Molecular characterization and immune modulation properties of Clonorchis sinensis-derived RNASET2. Parasit Vectors. 2013;6:360.

13. Xu Y, Lin J, Bian M, Chen W, Liang P, Wang X, et al. CsRNASET2 is an important component of Clonorchis sinensis responsible for eliciting Th2 immune response. Parasitol Res. 2015;114:2371-9.

14. Wang $Y$, Bai $X$, Zhu H, Wang $X$, Shi $H$, Tang $B$, et al. Immunoproteomic analysis of the excretorysecretory products of Trichinella pseudospiralis adult worms and newborn larvae. Parasit Vectors. 2017;10:579.

15. Bian M, Li S, Wang X, Xu Y, Chen W, Zhou C, et al. Identification, immunolocalization, and immunological characterization of nitric oxide synthase-interacting protein from Clonorchis sinensis. Parasitol Res. 2014;113:1749-57.

16. Trépo C, Chan HL, Lok A. Hepatitis B virus infection. Lancet. 2014;384:2053-63.

17. Bertoletti A, Ferrari C. Innate and adaptive immune responses in chronic hepatitis $B$ virus infections: towards restoration of immune control of viral infection. Postgrad Med J. 2013;89:294-304.

18. Ferrari C. HBV and the immune response. Liver Int. 2015;35 Suppl 1:121-8.

19. Meng Z, Chen Y, Lu M. Advances in Targeting the Innate and Adaptive Immune Systems to Cure Chronic Hepatitis B Virus Infection. Front Immunol. 2019;10:3127.

20. Boni C, Laccabue D, Lampertico P, Giuberti T, Viganò M, Schivazappa S, et al. Restored function of HBV-specific T cells after long-term effective therapy with nucleos(t)ide analogues. Gastroenterology. 2012;143:963-73.

21. Fisicaro P, Barili V, Rossi M, Montali I, Vecchi A, Acerbi G, et al. Pathogenetic Mechanisms of T Cell Dysfunction in Chronic HBV Infection and Related Therapeutic Approaches. Front Immunol. 2020;11:849. 
22. Ye B, Liu X, Li X, Kong H, Tian L, Chen Y. T-cell exhaustion in chronic hepatitis B infection: current knowledge and clinical significance. Cell Death Dis. 2015;6:e1694.

23. Saeidi A, Zandi K, Cheok YY, Saeidi H, Wong WF, Lee C, et al. T-Cell Exhaustion in Chronic Infections: Reversing the State of Exhaustion and Reinvigorating Optimal Protective Immune Responses. Front Immunol. 2018;9:2569.

24. Menne S, Tennant BC. Unraveling hepatitis B virus infection of mice and men (and woodchucks and ducks). Nat Med. 1999;5:1125-6.

25. Bertoletti A, Naoumov NV. Translation of immunological knowledge into better treatments of chronic hepatitis B. J Hepatol. 2003;39:115-24.

26. Chisari FV, Isogawa M, Wieland SF. Pathogenesis of hepatitis B virus infection. Pathol Biol (Paris). 2010;58:258-66.

27. Gallimore A, Glithero A, Godkin A, Tissot AC, Plückthun A, Elliott T, et al. Induction and exhaustion of lymphocytic choriomeningitis virus-specific cytotoxic $T$ lymphocytes visualized using soluble tetrameric major histocompatibility complex class I-peptide complexes. J Exp Med. 1998;187:1383-93.

28. Zajac AJ, Blattman JN, Murali-Krishna K, Sourdive DJ, Suresh M, Altman JD, et al. Viral immune evasion due to persistence of activated T cells without effector function. J Exp Med. 1998;188:2205-13.

29. Virgin HW, Wherry EJ, Ahmed R. Redefining chronic viral infection. Cell. 2009;138:30-50.

30. Evans A, Riva A, Cooksley H, Phillips S, Puranik S, Nathwani A, et al. Programmed death 1 expression during antiviral treatment of chronic hepatitis $\mathrm{B}$ : Impact of hepatitis $\mathrm{B}$ e-antigen seroconversion. Hepatology. 2008;48:759-69.

31. Ye B, Li X, Dong Y, Wang Y, Tian L, Lin S, et al. Increasing LAG-3 expression suppresses T-cell function in chronic hepatitis B: A balance between immunity strength and liver injury extent. Medicine (Baltimore). 2017;96:e5275.

32. Liu Y, Gao LF, Liang XH, Ma CH. Role of Tim-3 in hepatitis B virus infection: An overview. World J Gastroenterol. 2016;22:2294-303.

33. Wongjitrat C, Sukwit S, Chuenchitra T, Seangjaruk P, Rojanasang P, Romputtan P, et al. CTLA-4 and its ligands on the surface of T- and B-lymphocyte subsets in chronic hepatitis B virus infection. $J$ Med Assoc Thai. 2013;96 Suppl 1:S54-9.

34. Hartzell S, Bin S, Cantarelli C, Haverly M, Manrique J, Angeletti A, et al. Kidney Failure Associates With T Cell Exhaustion and Imbalanced Follicular Helper T Cells. Front Immunol. 2020;11:583702. 
35. Schietinger A, Greenberg PD. Tolerance and exhaustion: defining mechanisms of T cell dysfunction. Trends Immunol. 2014;35:51-60.

36. Wherry EJ, Kurachi M. Molecular and cellular insights into T cell exhaustion. Nat Rev Immunol. 2015;15:486-99.

37. Fisicaro P, Boni C, Barili V, Laccabue D, Ferrari C. Strategies to overcome HBV-specific T cell exhaustion: checkpoint inhibitors and metabolic re-programming. Curr Opin Virol. 2018;30:1-8.

38. Wherry EJ. T cell exhaustion. Nat Immunol. 2011;12:492-9.

39. Castellino F, Germain RN. Chemokine-guided CD4+ T cell help enhances generation of IL6RalphahighIL-7Ralpha high prememory CD8+ T cells. J Immunol. 2007;178:778-87.

40. Trautmann T, Kozik JH, Carambia A, Richter K, Lischke T, Schwinge D, et al. CD4+ T-cell help is required for effective CD8+ T cell-mediated resolution of acute viral hepatitis in mice. PLoS One. 2014;9:e86348.

41. Wherry EJ, Ahmed R. Memory CD8 T-cell differentiation during viral infection. J Virol. 2004;78:553545.

42. Antoine P, Varner V, Carville A, Connole M, Marchant A, Kaur A. Postnatal acquisition of primary rhesus cytomegalovirus infection is associated with prolonged virus shedding and impaired CD4+ T lymphocyte function. J Infect Dis. 2014;210:1090-9.

43. Lykken JM, DiLillo DJ, Weimer ET, Roser-Page S, Heise MT, Grayson JM, et al. Acute and chronic B cell depletion disrupts $\mathrm{CD} 4+$ and $\mathrm{CD} 8+\mathrm{T}$ cell homeostasis and expansion during acute viral infection in mice. J Immunol. 2014;193:746-56.

44. Li W, Dong H, Huang Y, Chen T, Kong X, Sun H, et al. Clonorchis sinensis Co-infection Could Affect the Disease State and Treatment Response of HBV Patients. PLoS Negl Trop Dis. 2016;10:e4806.

45. Blackburn SD, Shin H, Haining WN, Zou T, Workman CJ, Polley A, et al. Coregulation of CD8+ T cell exhaustion by multiple inhibitory receptors during chronic viral infection. Nat Immunol. 2009;10:29-37.

46. Nakamoto N, Cho H, Shaked A, Olthoff K, Valiga ME, Kaminski M, et al. Synergistic reversal of intrahepatic HCV-specific CD8 T cell exhaustion by combined PD-1/CTLA-4 blockade. PLoS Pathog. 2009;5:e1000313.

47. Kaufmann DE, Kavanagh DG, Pereyra F, Zaunders JJ, Mackey EW, Miura T, et al. Upregulation of CTLA-4 by HIV-specific CD4+ T cells correlates with disease progression and defines a reversible immune dysfunction. Nat Immunol. 2007;8:1246-54. 
48. Jin HT, Anderson AC, Tan WG, West EE, Ha SJ, Araki K, et al. Cooperation of Tim-3 and PD-1 in CD8 Tcell exhaustion during chronic viral infection. Proc Natl Acad Sci U S A. 2010;107:14733-8.

49. Shaw DM, Merien F, Braakhuis A, Dulson D. T-cells and their cytokine production: The antiinflammatory and immunosuppressive effects of strenuous exercise. Cytokine. 2018;104:136-42.

50. Lala V, Goyal A, Bansal P, Minter DA. Liver Function Tests. 2021.

\section{Figures}




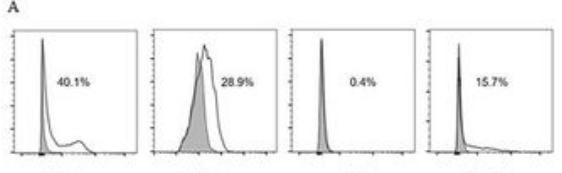

PQ.1 Tiм.3 CT:3
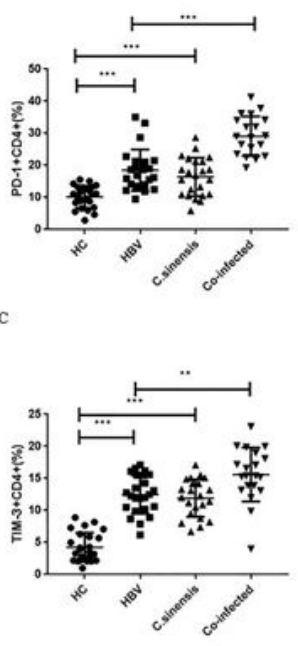

F

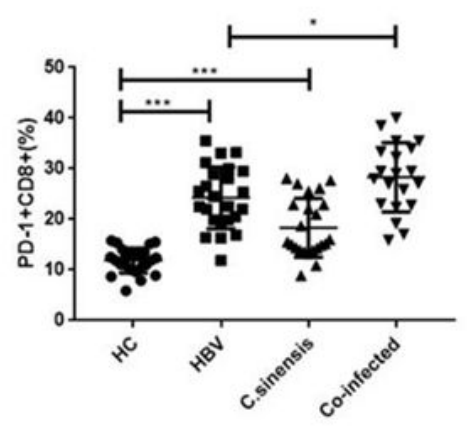

G

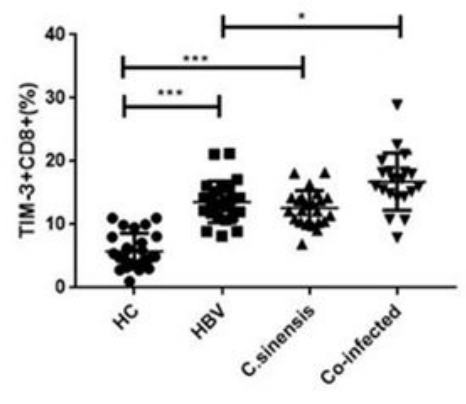

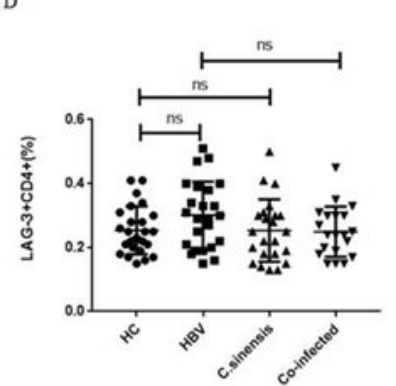

E

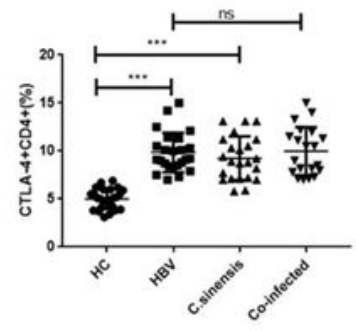

$\mathrm{H}$

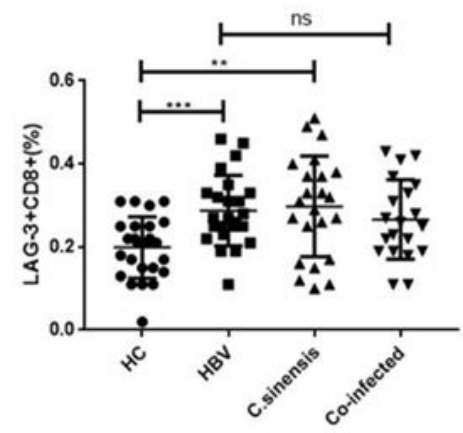

I

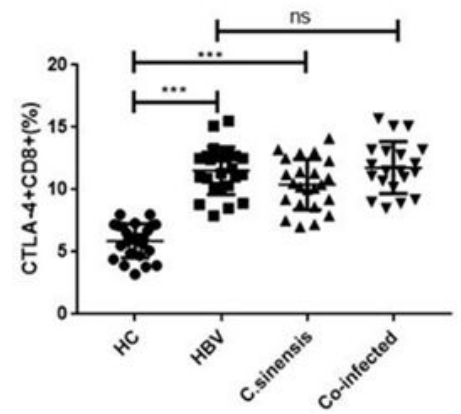

Figure 1

Gating strategy and expression profiles of PD-1,TIM-3, LAG-3 and CTLA-4 in CD4+T and CD8+T cells of in the co-infected group, HBV group, $C$. sinensis group and HC group. (A) The gating strategies and representative results of PD-1,TIM-3, LAG-3 and CTLA-4 expression in CD4+T and CD8+T cells. (B-I) The percentages of PD-1+CD4+(B), TIM-3+CD4+(C), LAG-3+CD4+(D), CTLA-4+CD4+(E), PD-1+CD8+(F), TIM3+CD8+(G), LAG-3+CD8+(H), CTLA-4+CD8+(I) T cells expression from co-infected group, HBV group, C. 
sinensis group and $\mathrm{HC}$ group. Data show the means \pm SD. Asterisks indicate statistically significant differences between Two groups, as measured by paired, two-tailed Student's t-test $\left({ }^{*} p<0.05,{ }^{*} p<0.01\right.$, $\star \star \star ~ p<0.001)$

A
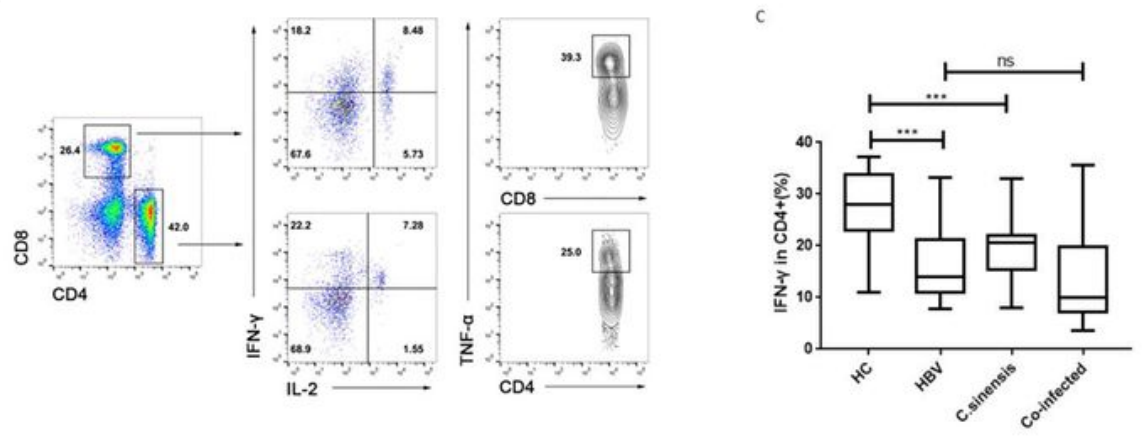

B

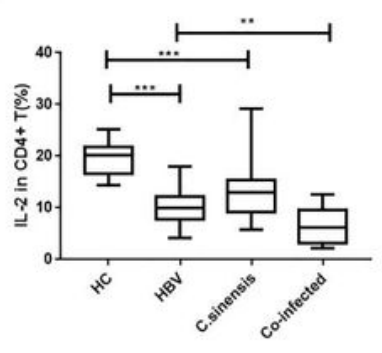

D
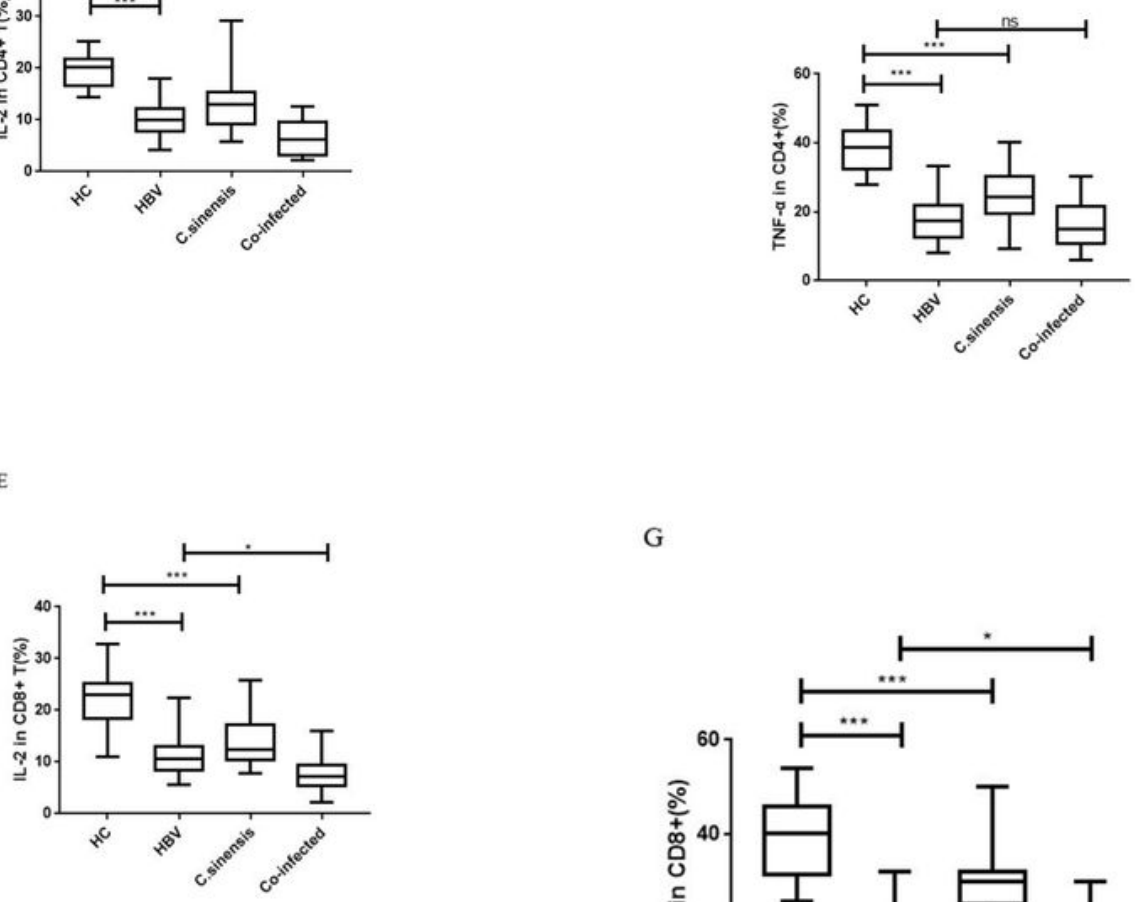

G
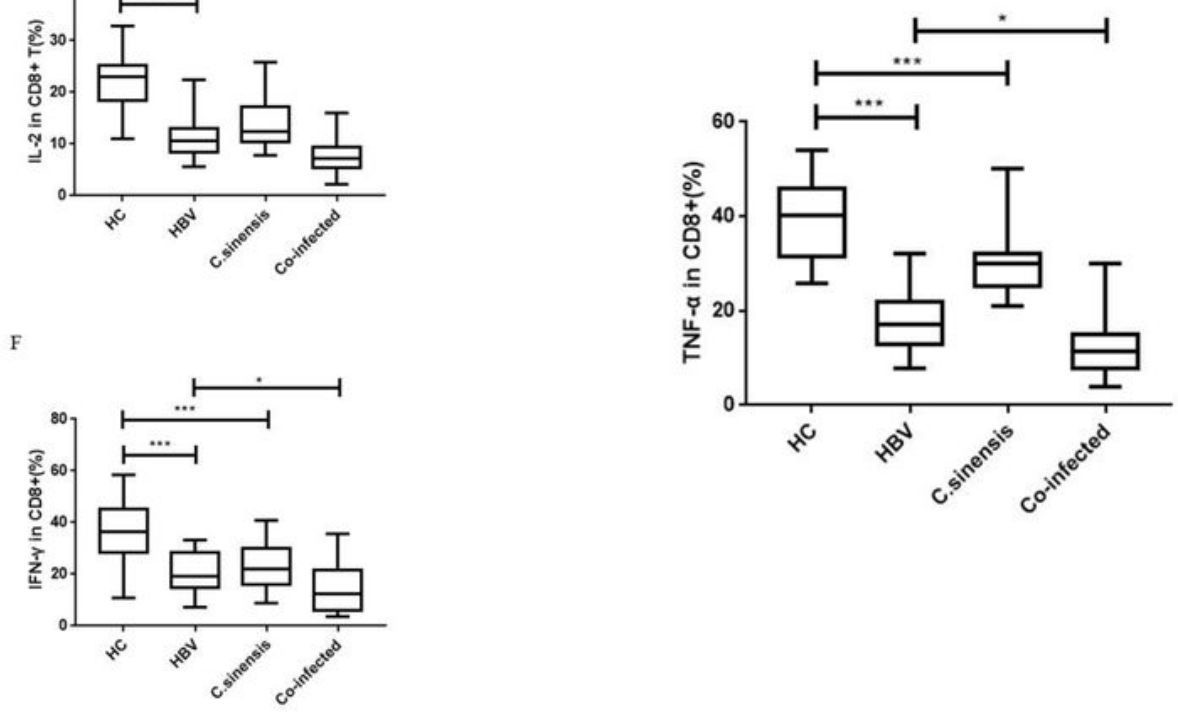

Figure 2

Gating strategy and expression profiles of IL-2, IFN- $\gamma$, and TNF- $\alpha$ in CD4+T and CD8+T cells of in the coinfected group, HBV group, $\mathrm{C}$. sinensis group and HC group. (A) The gating strategies and representative 
results of IL-2, IFN-y, and TNF- $\alpha$ expression in CD4+T and CD8+T cells. (B-G) The percentages of IL-2 in CD4+(B), IFN- $\gamma$ in CD4+(C), TNF- $\alpha$ in CD4+(D), IL-2 in CD8+(E), IFN- $\gamma$ in CD8+(F), TNF- $\alpha$ in CD8+(G) T cells expression from co-infected group, HBV group, $\mathrm{C}$. sinensis group and $\mathrm{HC}$ group. Data show the means $\pm S D$. Asterisks indicate statistically significant differences between Two groups, as measured by paired, two-tailed Student's t-test $\left({ }^{\star} p<0.05, * \star p<0.01,{ }^{\star * *} p<0.001\right)$
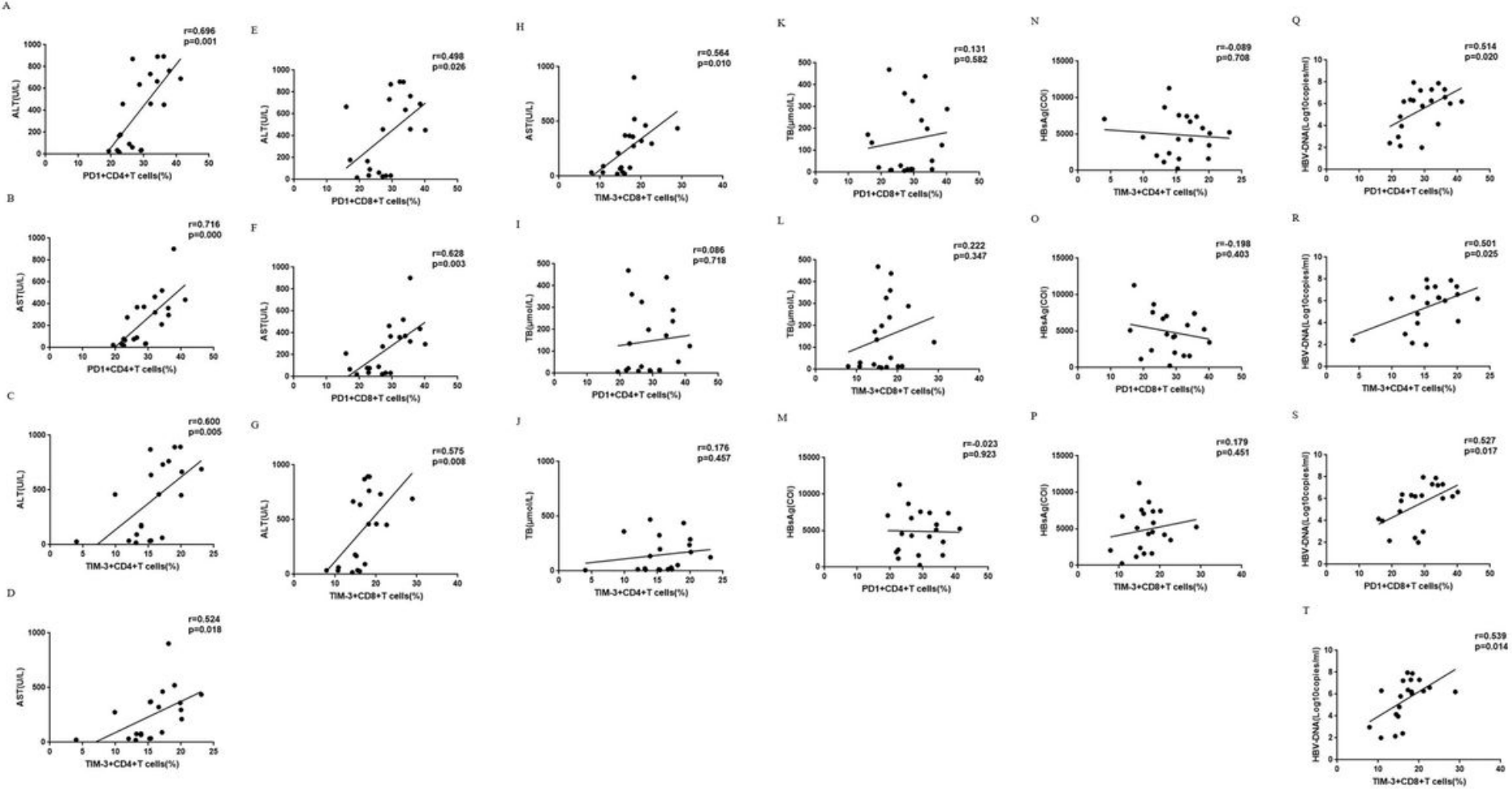

\section{Figure 3}

Correlation between the percentages of PD-1+and TIM-3+ on the CD4+T cells and CD8+T cells and the levels of ALT, AST, TB, HBsAg and HBV DNA in the co-infected patients. Relationship between the serum ALT levels and the percentages of PD-1+CD4+(A), TIM-3+CD4+(C), PD-1+CD8+(E), TIM-3+CD8+(G) T cells expression in the co-infected patients; The relationship between the serum AST levels and the percentage of PD-1+CD4+(B), TIM-3+CD4+(D), PD-1+CD8+(F), TIM-3+CD8+(H) T cells expression in the co-infected patients; The relationship between the TB levels and the percentages of PD- $1+C D 4+(I), T I M-3+C D 4+(J)$, PD-1+CD8+(K), TIM-3+CD8+(L) T cells expression in the co-infected patients; The relationship between the HBsAg levels and the percentages of PD- $1+C D 4+(M), T I M-3+C D 4+(N), P D-1+C D 8+(0), T I M-3+C D 8+(P)$ $T$ cells expression in the co-infected patients; The relationship between the HBV DNA levels and the percentages of PD- $1+C D 4+(\mathrm{Q})$, $\mathrm{TIM}-3+\mathrm{CD} 4+(\mathrm{R}), \mathrm{PD}-1+\mathrm{CD} 8+(\mathrm{S}), \mathrm{TIM}-3+\mathrm{CD} 8+(\mathrm{T}) \mathrm{T}$ cells expression in the co-infected patients.

\section{Supplementary Files}

This is a list of supplementary files associated with this preprint. Click to download. 
- GraphicalAbstract.png

Page 19/19 